\title{
Manejo de tumores gástricos del estroma gastrointestinal encontrados en cirugía bariátrica: un desafío quirúrgico
}

\author{
Management of gastrointestinal stromal tumors encountered during \\ bariatric surgery: a surgical challenge \\ Carlos Luna Jaspe1, Rubén Luna², Luis Felipe Cabrera³, Raúl Suárez³ ${ }^{3}$ Diego Salcedo³ \\ 1 Jefe, Servicio de Cirugía Bariátrica y Metabólica, Clínica Universitaria El Bosque, Bogotá, D.C., Colombia \\ 2 Cirujano bariátrico, Clínica Universitaria El Bosque, Bogotá, D.C., Colombia \\ Residente de Cirugía General, Clínica Universitaria El Bosque, Bogotá, D.C., Colombia
}

\section{Resumen}

Introducción. Se han reportado hallazgos incidentales durante la cirugía bariátrica, como tumores gástricos (o,6 a o,8 \%), entre los cuales el más frecuente es el del estroma gastrointestinal (GIST). Es importante tener en cuenta que el pilar del tratamiento de este tipo de tumores es la cirugía con márgenes negativos.

Métodos y resultados. Se llevó a cabo un estudio retrospectivo, descriptivo y observacional, desde enero de 2013 hasta junio de 2016; durante este periodo, 320 pacientes fueron sometidos a cirugía bariátrica, manga gástrica en 250 y derivación gástrica en 70, en la Clínica Universitaria El Bosque. En tres de ellos, dos con manga gástrica y uno con derivación gástrica, se encontró incidentalmente un tumor gástrico del estroma gastrointestinal (GIST) con confirmación por histopatología; mediante escisión, se obtuvieron bordes negativos para tumor, no se presentaron complicaciones ni se prolongó la estancia hospitalaria.

Discusión y conclusiones. Teniendo en cuenta la ubicación de la lesión, es posible la intervención laparoscópica de los tumores gástricos del estroma gastrointestinal de $2 \mathrm{~cm}$ o más, encontrados durante un procedimiento de cirugía bariátrica.

Palabras clave: tumores del estroma gastrointestinal; estómago; obesidad; cirugía bariátrica; derivación gástrica; laparoscopía.

\footnotetext{
Abstract

Introduction: Incidental findings are reported in bariatric surgery, such as gastric tumors in $2 \%$ to $8 \%$, gastrointestinal stromal tumors (GIST) being the most frequent. The pillar of handling this type of tumor is surgery with negative margins.

Methods and Results: This is a descriptive retrospective study covering the period January 2013 to June 2016 during which 320 patients underwent bariatric surgery, 250 gastric sleeve and 70 gastric bypass, among which three had the incidental finding of gastric GIST confirmed by pathology; two patients had gastric sleeve and one gastric bypass; resection of the tumor was carried out obtaining adequate negative margins with no morbidities and no prolonged hospital stay.

Discussion and Conclusions: Gastric sleeve and gastric bypass are the preferred surgical options in bariatric surgery, allowing resection of the incidentally found gastrointestinal stromal tumors for curative treatment, without altering the outcome or purpose of the bariatric surgery procedure.

Keywords: gastrointestinal stromal tumors; stomach; obesity; bariatric surgery; gastric bypass; laparoscopy.

Fecha recibido: 09/06/2017 - Fecha aceptación: 06/10/2017

Correspondencia: Luis Felipe Cabrera Vargas, MD, Bogotá, D.C., Colombia

Correo electrónico: luis.felipe.cabrera@hotmail.com

Citar como: Luna-Jaspe C, Luna R, Cabrera LF, Suárez R, Salcedo D. Manejo de tumores gástricos del estroma gastrointestinal encontrados en cirugía bariátrica: un desafío quirúrgico. Rev Colomb Cir. 2018;33:173-80. https://doi.org/10.30944/20117582.59
} 


\section{Introducción}

En los Estados Unidos, el 65 \% de la población sufre sobrepeso (índice de masa corporal mayor de $25 \mathrm{~kg} / \mathrm{m}^{2}$ ) y el número de cirugías bariátricas se ha incrementado notoriamente, de 16.000 en la década de los 90 a I03.00o en el 2013. De tal forma, la obesidad, como epidemia mundial, ha hecho que los procedimientos bariátricos se conviertan en una de las cirugías gastrointestinales más frecuentes actualmente, lo cual ha generado un aumento en el número de hallazgos incidentales intraoperatorios ${ }^{\mathrm{I}}$.

A pesar de los múltiples exámenes previos a la cirugía bariátrica, se reportan hallazgos quirúrgicos incidentales como el de tumores gástricos en o, 6 a o,8 \% de los casos ${ }^{2-4}$; entre estos, los más frecuentes son los tumores del estroma gastrointestinal (GIST). Este tipo de neoplasias tienen una incidencia de 6,8 casos por millón de habitantes, su ubicación más frecuente es el estómago, y deben diferenciarse de los leiomiomas y los schwanomas 5 .

Es importante tener en cuenta que el pilar del tratamiento de este tipo de tumores es su extirpación quirúrgica. Se considera que todo tumor del estroma gastrointestinal tiene potencial maligno y los de más de $2 \mathrm{~cm}$ han demostrado un mayor riesgo de diseminación. El tratamiento estándar es la resección oncológica sin linfadenectomía, ya que la diseminación ganglionar es nula. Sin embargo, no se excluye la posibilidad de que produzcan metástasis peritoneales o hepáticas, que no son infrecuentes, sobre todo en centros con gran casuística ${ }^{6}$.

En este estudio se presenta una serie de $3 \mathrm{ca}-$ sos de tumor del estroma gastrointestinal como hallazgo incidental en la cirugía bariátrica, de un grupo de 320 pacientes intervenidos por obesidad, se hace una revisión de la literatura científica y se propone un algoritmo de manejo

\section{Materiales y métodos}

Se llevó a cabo un estudio retrospectivo, descriptivo y observacional, desde enero del 2013 hasta junio del 20I6. Durante este periodo, 320 pacientes con un índice de masa corporal (IMC) de 40 $\mathrm{kg} / \mathrm{m}^{2}$ o mayor, o con un IMC de 35 a $40 \mathrm{~kg} / \mathrm{m}^{2}$ con al menos una comorbilidad relacionada con la obesidad, fueron sometidos a cirugía bariátrica: 250 a manga gástrica y 70 a derivación gástrica, en la Clínica Universitaria El Bosque.

De estos pacientes, se incluyeron en este estudio tres $(0,9 \%)$ con hallazgo quirúrgico de tumor del estroma gastrointestinal, dos sometidos a manga gástrica y uno a derivación gástrica. La manga gástrica es la técnica estándar en cirugía bariátrica de nuestra institución en pacientes con IMC de 35 a $40 \mathrm{~kg} / \mathrm{m}^{2}$ con, al menos, una comorbilidad, y en aquellos con IMC de $40 \mathrm{~kg} / \mathrm{m}^{2}$ o más sin diabetes mellitus; la derivación gástrica se indica según el tamaño de la lesión y su ubicación.

En los tres casos incluidos con hallazgo incidental de tumor submucoso, el diagnóstico de tumor del estroma gastrointestinal se confirmó por histopatología con inmunohistoquímica (CD-II7, CD-34 y DOG-I).

Los pacientes se sometieron a una endoscopia gastrointestinal superior preoperatoria de rutina. Durante la laparoscopia, el estómago se inspeccionó macroscópicamente para detectar cualquier tipo de lesión tumoral, al igual que al extraer la pieza quirúrgica de forma manual, como en los pacientes sometidos a manga gástrica, y se revisaron los informes de patología para detectar neoplasias en el estómago resecado.

De los pacientes incluidos, se obtuvieron los siguientes datos: edad, sexo, IMC, comorbilidades, hallazgos intraoperatorios, tipo de cirugía bariátrica y resultado oncológico de patología. Las características tumorales registradas incluyeron el tipo de tumor, el tamaño, la localización, el estado de los márgenes, la tasa mitótica y la inmunohistoquímica; también, se observó el seguimiento posoperatorio.

En dos de los casos, la presencia del tumor no requirió una variación de la técnica para obtener márgenes negativos; en el otro, por el hallazgo intraoperatorio del tumor se canceló el procedimiento quirúrgico inicial de manga gástrica y se practicó una tomografía computadorizada (TC) con contraste antes de la nueva intervención, que consistió en una derivación gástrica. A es- 
tos pacientes se les hizo seguimiento oncológico con TC abdominal con contraste cada seis meses durante 5 años y, después, anualmente.

La aprobación del estudio fue otorgada por la Junta de Ética de la institución. Todos los pacientes firmaron el consentimiento informado para manga gástrica o derivación gástrica como alternativa en caso de hallazgos inesperados, como tumores, grasa visceral masiva o adherencias graves, o de cambios de su estado clínico.

\section{Resultados}

En este estudio se incluyeron dos mujeres y un hombre. La edad media fue de 55 años y, el IMC promedio, de $42 \mathrm{~kg} / \mathrm{m}^{2}$. Los tres pacientes sufrían hipertensión arterial sistémica y apnea del sueño, y uno presentaba diabetes mellitus de tipo 2. Ninguno tenía enfermedades hereditarias relacionadas con tumores del estroma gastrointestinal, como la neurofibromatosis de tipo I. El tiempo quirúrgico promedio fue de 60 minutos y la estancia hospitalaria fue de dos días en todos ellos. No presentaron complicaciones o muerte y el sangrado intraoperatorio no aumentó con respecto a un procedimiento bariátrico sin hallazgos tumorales incidentales; fue de Io $\mathrm{ml}$, aproximadamente.

En los tres pacientes, la cirugía bariátrica escogida era la manga gástrica debido a las comorbilidades y el IMC. En los dos primeros se mantuvo esta opción. En el tercero, durante la laparoscopia se encontró una lesión submucosa en la cara anterior del cuerpo gástrico, por lo cual se canceló la intervención y se tomó una TC con la cual se descartó metástasis a distancia; se consideró que la lesión podía resecarse y, por su ubicación y tamaño $(8 \mathrm{~cm})$, se decidió practicar una derivación gástrica con inclusión de la lesión en el área de resección durante la laparoscopia, sin necesidad de conversión a cirugía abierta.

El tipo de cirugía bariátrica se decidió según la ubicación del tumor del estroma gastrointestinal; en los dos pacientes con manga gástrica, el tumor se ubicó en la curvatura mayor y el fondo gástrico y, en el otro, en la cara anterior del cuerpo gástrico, por lo que se optó por la derivación gástrica con el fin de obtener márgenes libres de tumor y llevar a cabo en el mismo tiempo quirúrgico la cirugía bariátrica y la oncológica (figura I).

Es importante resaltar que todas las piezas quirúrgicas extraídas en nuestro grupo de cirugía bariátrica y metabólica, son revisadas externa e internamente por el cirujano en el acto operatorio y, después, se someten a estudio histopatológico. El tamaño tumoral promedio fue de $3,5 \mathrm{~cm}$ con un máximo de $8 \mathrm{~cm}$, y la localización más frecuente fue la curvatura mayor. Los márgenes quirúrgicos fueron negativos para compromiso tumoral en los tres especímenes. Todos los tumores presentaron marcadores positivos para DOG-I, CD-34 y CD-II7 (figura 2).

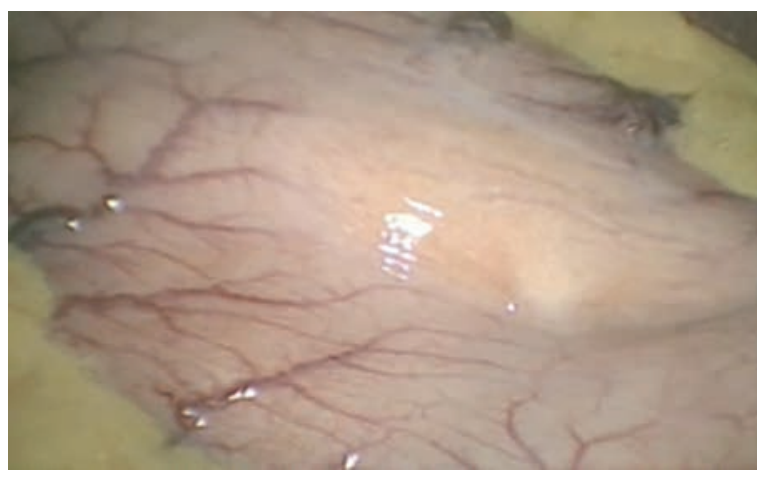

Figura 1. Tumor del estroma gastrointestinal hacia la curvatura mayor del estómago identificado en la laparoscopia

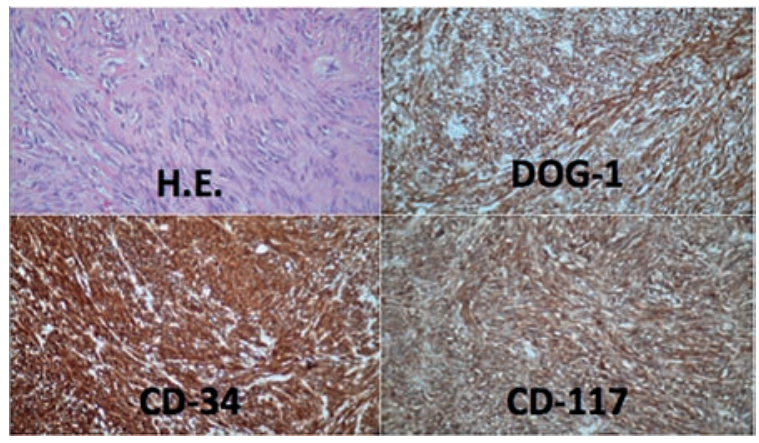

Figura 2. Inmunohistoquímica de las piezas quirúrgicas con marcadores positivos para DOG-1, CD-34 y CD117 , indicativos de tumor del estroma gastrointestinal 
Los tres pacientes evolucionaron de forma favorable en el posoperatorio y se siguió el protocolo establecido, dando el alta hospitalaria al segundo. Ninguno requirió tratamiento adyuvante con quimioterapia con imatinib, ya que se clasificaron como de riesgo intermedio, bajo y muy bajo riesgo, con base en un bajo grado histológico, un índice mitótico bajo, la ubicación gástrica y los bordes quirúrgicos negativos; solo un paciente tenía un tumor de más de $5 \mathrm{~cm}$, pero con índice mitótico bajo. El seguimiento oncológico se hizo con TC abdominal con contraste cada seis meses durante cinco años y, después, anualmente. No se presentó recaída tumoral local o a distancia, es decir, los tres continuaron libres de enfermedad (tabla I).

\section{Discusión}

Los del estroma son los tumores mesenquimales gastrointestinales más frecuentes. Se originan en las células de Cajal que se ubican en el plexo mientérico, y su inmunohistoquímica expresa
c-KIT (95\%), proteína cinasa (8o \%) y CD 34 $(60-70 \%)^{4}$. Su ubicación más común es en el estómago (50 a $60 \%$ ) y son más infrecuentes en el esófago (5\%). En el estómago, se presentan más frecuentemente en el fondo y en el cardias; sin embargo, en esta serie dos se presentaron en la curvatura mayor y uno en el cuerpo gástrico 5 . Su incidencia varía de 6,5 a I4,5 casos por millón de habitantes ${ }^{6}$.

Se encuentran asociados a enfermedades hereditarias, como la neurofibromatosis de tipo I o la tríada de Carney, aunque en los presentes casos ninguno tenía antecedentes de este tipo de enfermedades genéticas .

$\mathrm{Su}$ incidencia como hallazgo incidental durante la cirugía bariátrica $(0,9 \%)$ concordó con otros reportes (o,6 a o,8 \%), como el de Yuval, et al., de 827 pacientes sometidos a manga gástrica, y el de Sánchez, et al., de 517 pacientes sometidos a derivación gástrica; es más frecuente durante la derivación gástrica, con respecto a la población general $(0,0006 \%)^{8-10}$.

Tabla 1. Características demográficas de los pacientes

\begin{tabular}{lccc}
\hline & $\mathbf{1}$ & Paciente & $\mathbf{3}$ \\
\hline Edad (años) & 56 & $\mathbf{2}$ & 54 \\
Sexo & Mujer & Mujer & Hombre \\
Tamaño (cm) & 2 & 2 & 8 \\
Ubicación & Curvatura mayor & Curvatura mayor & Cara anterior del \\
Índice mitótico & 0 & 0 & cuerpo gástrico \\
Grado histológico & Bajo & Bajo & Bajo \\
Cirugía & Manga gástrica & Manga gástrica & Bypass gástrico \\
Tiempo quirúrgico (min) & 45 & 45 & 90 \\
Sangrado (ml) & 10 & 10 & 10 \\
Complicaciones & No & No & No \\
Estancia hospitalaria (días) & 2 & 2 & 2 \\
Bordes quirúrgicos & Negativos & Negativos & Negativos \\
Marcadores DoG 1, CD34, & Positivos & Positivos & Positivos \\
CD117 & No & No & No \\
Imatinib & Sin recaídas & Sin recaídas & Sin recaídas \\
Seguimiento & & &
\end{tabular}


Típicamente, los tumores del estroma gastrointestinal afectan adultos mayores, con un promedio de edad de 60 a 65 años, son relativamente raros antes de los 40 años y menos del I \% ocurre en menores de 2I años; no muestran predilección según el sexo ${ }^{\mathrm{II}, \mathrm{I2}}$.

En esta serie, a ninguno de los pacientes se le practicaron pruebas para detectar Helicobacter pylori, pues no se ha demostrado una asociación entre esta bacteria y el desarrollo de este tipo de tumor ${ }^{13,14}$. Todos los tumores del estroma gastrointestinal expresan CD-II7, el cual es un producto del gen C-KIT y se coexpresa con DOG-I (una proteína de función desconocida), lo cual se reflejó en su inmunohistoquímica en la presente serie ${ }^{15,16}$. Los distinguen de otras neoplasias mesenquimales gastrointestinales, como leiomiomas y neuromas, los cuales se originan en el plexo mientérico de Auerbach y cuya incidencia corresponde a 0,2 \% de todos los tumores gástricos de comportamiento benigno y presentan transformación maligna cuando su diámetro es de $5 \mathrm{~cm}$ o mayor ${ }^{16-18}$.

El curso clínico de estos tumores, en general, es asintomático y se ha reportado que pueden manifestarse inicialmente con sangrado gastrointestinal alto, masa palpable o síndrome pilórico, cuando su tamaño es mayor de 2 a $5 \mathrm{~cm}$. Sin embargo, a pesar de que uno de nuestro pacientes presentaba un tumor de $8 \mathrm{~cm}$, nunca presentó sintomatología gastrointestinal asociada, posiblemente debido a su ubicación en la cara anterior del cuerpo gástrico, y no se evidenció en la endoscopia preoperatoria, probablemente por su ubicación submucosa ${ }^{19,20}$.

El tratamiento ideal para este tipo de tumores es la resección quirúrgica sin linfadenectomía, con los siguientes principios quirúrgicos: respetar la seudocápsula, evitar la ruptura del tumor y lograr una resección $R_{o}$ con bordes macroscópicos libres, lo cual se puede lograr durante la cirugía bariátrica, según la ubicación, incluyendo el tumor en el área de resección.

De esta forma, cuando el procedimiento quirúrgico es una manga gástrica, se pueden obtener buenos resultados oncológicos si se encuentra en la curvatura mayor o el fondo gástrico; de lo contrario, se recomienda la derivación gástrica para tratar la obesidad y el tumor haciendo una resección con bordes macroscópicamente negativos, sin necesidad de una gastrectomía total del remanente. Esta se recomienda en presencia de enfermedades asociadas que requieran de vigilancia endoscópica periódica, como pólipos gástricos, úlceras o metaplasia intestinal.

Cabe resaltar que, con la derivación gástrica, en esta serie, se hizo el tratamiento bariátrico y oncológico, de los pacientes con tumor del estroma gastrointestinal incidental, sin cambios en el tiempo quirúrgico, el sangrado intraoperatorio o el tiempo de hospitalización establecidos en el protocolo de este centro de excelencia para manejo quirúrgico de la obesidad ${ }^{21-23}$. Sin embargo, si el tumor se ubica en la unión esofagogástrica, el procedimiento adecuado sería una gastrectomía total con reconstrucción en Y de Roux y, si su tamaño es menor de $2 \mathrm{~cm}$, el paciente se beneficiaría del seguimiento endoscópico y tomográfico ${ }^{24,25}$.

El manejo quirúrgico de los tumores del estroma gastrointestinal menores de $2 \mathrm{~cm}$ es controversial. Según las guías del National Comprehensive Cancer Network (NCCN), en todo tumor gástrico del estroma gastrointestinal con menos de 50 mitosis por campo de mayor aumento y con un tamaño menor de $2 \mathrm{~cm}$, el riesgo de progresión de la enfermedad es cero y, por lo tanto, no se requiere de tratamiento quirúrgico. Sin embargo, en las guías canadienses basadas en el estudio de Huang, et al., sobre recurrencia tumoral y riesgo de metástasis, en el cual el Io \% de los pacientes con tumores del estroma gastrointestinal clasificados como de muy bajo riesgo presentaron metástasis, se recomienda el manejo quirúrgico de entrada ${ }^{24,26,27}$.

Es importante tener en cuenta que este tipo de tumores están subdiagnosticados, pues los menores de $2 \mathrm{~cm}$ pueden pasar desapercibidos para el cirujano durante la intervención. Sin embargo, en una serie de Crouthamel, et al., de I.4I5 cirugías bariátricas, de 205 piezas quirúrgicas enviadas a patología, solo se encontró una lesión tumoral que pasó inadvertida por el cirujano 
y que correspondió a un leiomioma de $2 \mathrm{~mm}$. Además, las guías del NCCN para el manejo de los tumores gástricos del estroma gastrointestinal, recomiendan solo seguimiento endoscópico para aquellos menores de $2 \mathrm{~cm}$ debido a su curso benigno.

Miettinen, et al., en un estudio de seguimiento a largo plazo de I.765 pacientes con tumores del estroma gastrointestinal menores de $2 \mathrm{~cm}$, sin recurrencia, progresión de la enfermedad o mortalidad asociada, recomiendan que el cirujano haga un examen macroscópico rutinario, tanto externo como interno, de las piezas quirúrgicas extraídas, y que todo el tejido resecado se someta a estudio histopatológico, tal como se hace en nuestro centro para el manejo quirúrgico de la obesidad $^{28-30}$.

Este estudio presenta algunas limitaciones como ser realizado en un solo centro y que el diagnóstico intraoperatorio dependió de la perspectiva individual del cirujano. Sin embargo, cabe recalcar que todas las endoscopias preoperatorias de los pacientes fueron normales y que en los protocolos de cirugía bariátrica a nivel mundial, al igual que en nuestro grupo, no se incluyen la TC con contraste ni la resonancia magnética abdominales como estudios prequirúrgicos.

El seguimiento se debe llevar a cabo según las recomendaciones de las guías de la NCCN, es decir, con TC abdominal con contraste cada seis meses durante cinco años y después anualmente, para los pacientes de riesgo intermedio o alto, tal como se hizo en el paciente sometido a derivación gástrica en esta serie; sin embargo, a los pacientes clasificados como de muy bajo riesgo se les puede hace un seguimiento más espaciado, como en los dos sometidos a manga gástrica. Con lo anterior, nos permitimos proponer un algoritmo de manejo para este tipo de tumores hallados de forma incidental durante la cirugía bariátrica (figura 3) ${ }^{31}$.

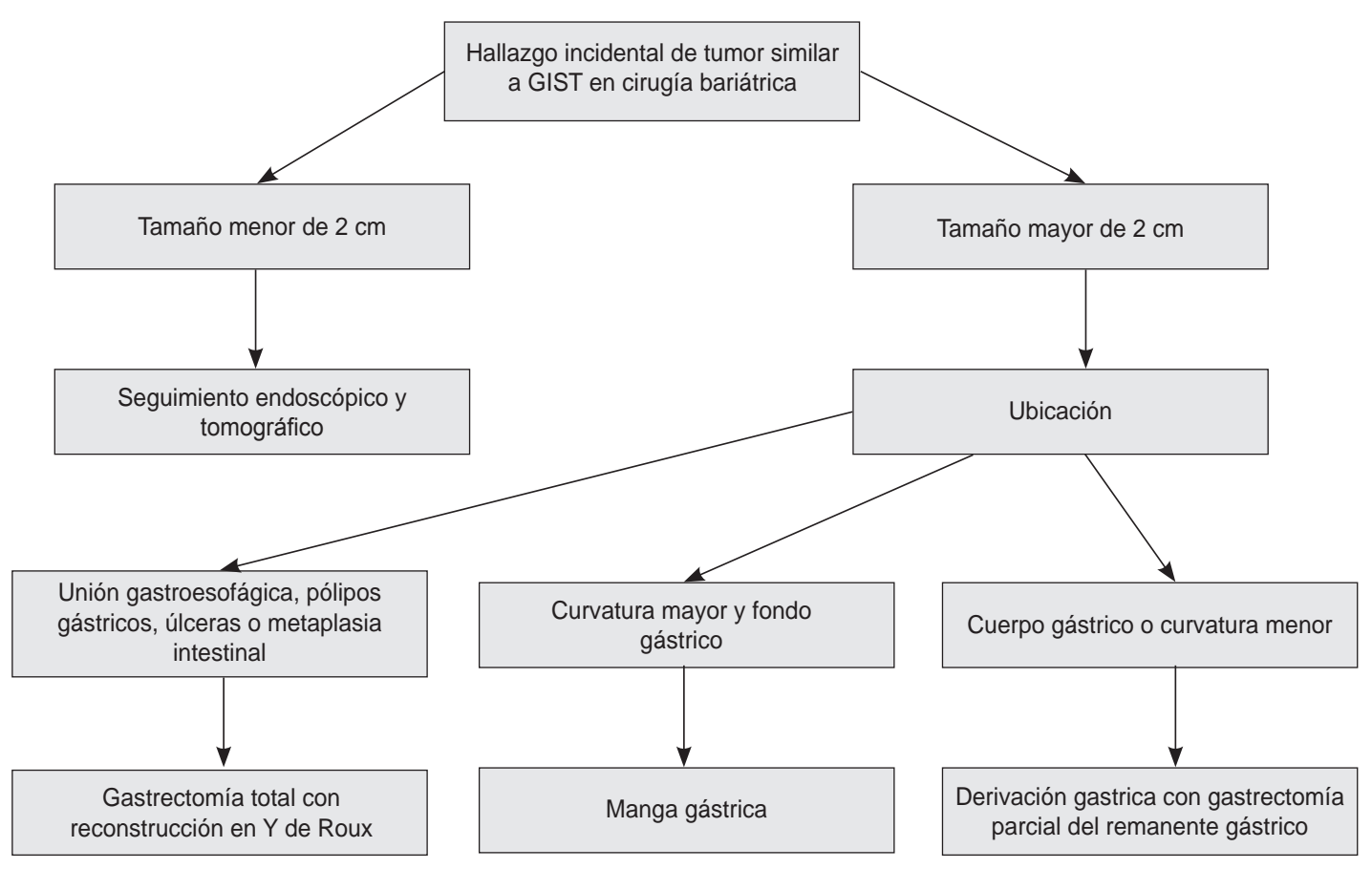

Figura 3. Esquema de manejo quirúrgico para tumores del estroma gastrointestinal (GIST) en cirugía bariátrica 


\section{Conclusiones}

El manejo laparoscópico de los tumores del estroma gastrointestinal de 2 o más $\mathrm{cm}$ de diámetro encontrados durante un procedimiento de cirugía bariátrica, es posible teniendo en cuenta su ubicación, obteniendo márgenes libres de enfermedad tumoral, baja morbilidad, ausencia de mortalidad, sin aumento en la estancia hospitalaria y garantizando una supervivencia libre de enfermedad en todos los pacientes.

Conflicto de intereses. Los autores no tienen potencial conflicto de intereses con respecto a la publicación de este documento.

\section{Referencias}

I. Chiappetta S, Theodoridou S, Stier C, Weiner R. Incidental finding of GIST during obesity surgery. Obes Surg. 2015;25:579-83.

2. Nguyen NT, Nguyen B, Gebhart A, Hohmann S. Changes in the makeup of bariatric surgery: A national increase in use of laparoscopic sleeve gastrectomy. J Am Coll Surg. 2013;216:252-7.

3. Tran T, Dávila JA, El-Serag HB. The epidemiology of malignant gastrointestinal stromal tumors: An analysis of I,458 cases from 1992 to 2000. Am J Gastroenterol. 2005;IOO:I62-8.

4. Yuval JB, Khalaileh A, Abu-Gazala M, Shachar Y, Keidar A, Mintz Y, et al. The true incidence of gastric GIST-a study based on morbidly obese patients undergoing sleeve gastrectomy. Obes Surg. 2014;24:2134-7.

5. Sánchez BR, Morton JM, Curet MJ, Alami RS, Safadi BY. Incidental finding of gastrointestinal stromal tumors (GISTs) during laparoscopic gastric bypass. Obes Surg. 2005;15:1384-8.

6. Crouthamel M, Kaufman J, Billing J, Billing P, Landerholm R. Incidental gastric mesenchymal tumors identified during laparoscopic sleeve gastrectomy. Surg Obes Relat Dis. 2015;II:IO25-28.

7. Finnell CW, Madan AK, Ternovits CA, Menachery SJ, Tichansky DS. Unexpected pathology during laparoscopic bariatric surgery. Surg Endosc. 2007;21:867-9.

8. Blay JY, von Mehren M, Blackstein ME. Perspective on updated treatment guidelines for patients with gastrointestinal stromal tumors. Cancer. 20I0;II6:5126-37.

9. Greenbaum D, Friedel D. Unanticipated findings at bariatric surgery. Surg Obes Relat Dis. 2005;I:22-4.

Io. Demetri GD, Benjamin RS, Blanke CD, Blay JY, Casali P, Choi H, et al. NCCN Task Force report: Management of patients with gastrointestinal stromal tumor (GIST) - update of the NCCN clinical practice guidelines. J Natl Compr Cancer Netw. 2007;5(Suppl.2):SI-29.
II. Demetri GD, von Mehren M, Antonescu CR, DeMatteo RP, Ganjoo KN, Maki RG, et al. NCCN Task Force report: Update on the management of patients with gastrointestinal stromal tumors. J Natl Compr Cancer Netw. 20I0;8(Suppl.2):SI-4I.

I2. Steigen SE, Eide TJ. Gastrointestinal stromal tumors (GISTs): A review. APMIS. 2009;II7:73-86.

I3. Tryggvason G, Gislason HG, Magnusson MK, Jonasson J. Gastrointestinal stromal tumors in Iceland, 19902003: The Icelandic GIST study, a population-based incidence and pathologic risk stratification study. Int J Cancer. 2005;II7:289-93.

I4. Fletcher CDM, Berman JJ, Corless C, Gorstein F, Lasota J, Longley BJ, et al. Diagnosis of gastrointestinal stromal tumors: A consensus approach. Hum Pathol. 2002;33:459-65.

I5. Goettsch WG, Bos SD, Breekveldt-Postma N, Herings RM, Hogendoorn PC. Incidence of gastrointestinal stromal tumors is underestimated: Results of a nation-wide study. Eur J Cancer. 2005;41:2868-72.

I6. Zheng L, Wu X, Kreis ME, Yu Z, Feng L, Chen C, et al. Clinicopathological and immunohistochemical characterisation of gastric schwannomas in 29 cases. Gastroenterol Res Pract. 20I4;20I4:202960. doi: IO.II55/2014/202960.

17. Rubio J, Marcos-Gragera R, Ortiz MR, Miró J, Vilardell L, Gironès J, et al. Population-based incidence and survival of gastrointestinal stromal tumors (GIST) in Girona, Spain. Eur J Cancer. 2007;43:I44-8.

I8. Mullady DK, Tan BR. A multidisciplinary approach to the diagnosis and treatment of gastrointestinal stromal tumor. J Clin Gastroenterol. 2013;47:578-85.

19. Nilsson B, Bümming P, Meis-Kindblom JM, Odén A, Dortok A, Gustavsson B, et al. Gastrointestinal stromal tumors: The incidence, prevalence, clinical course, and prognostication in the preimatinib mesylate era-a population based study in western Sweden. Cancer. 2005;IO3:82I-9.

20. National Comprehensive Cancer Network (NCCN). NCCN Clinical Practice Guidelines in Oncology. Soft tissue sarcoma Version 2.20I4. National Comprehensive Cancer Network. Fecha de consulta: 20 de junio de 20I7. Disponible en: http://www.nccn. org/professionals/physician_gls/pdf/sarcoma.pdf.

2I. Scherübl H, Faiss S, Knoefel WT, Wardelmann E. Management of early asymptomatic gastrointestinal stromal tumors of the stomach. World J Gastrointest Endosc. 2014;6:266-7I.

22. Agaimy A, Vassos N, Croner RS. Gastrointestinal manifestations of neurofibromatosis type I (Recklinghausen's disease): Clinicopathological spectrum with pathogenetic considerations. Int J Clin Exp Pathol. 2OI2;5:852-62.

23. Fong Y, Coit DG, Woodruff JM, Brennan MF. Lymph node metastasis from soft tissue sarcoma in adults. 
Analysis of data from a prospective database of $\mathbf{1 7 7 2}$ sarcoma patients. Ann Surg. 1993;217:72-7.

24. Blackstein ME, Blay JY, Corless C, Driman DK, Riddell $\mathrm{R}$, Soulières $\mathrm{D}$, et al. Canadian advisory committee on GIST gastrointestinal stromal tumours: Consensus statement on diagnosis and treatment. Can J Gastroenterol. 2006;20:157-63.

25. Buchwald H, Oien DM. Metabolic/bariatric surgery worldwide 20II. Obes Surg. 2013;23:427-36.

26. Raghavendra RS, Kini D. Benign, premalignant, and malignant lesions encountered in bariatric surgery. JSLS. 2012;16:360-72.

27. Yun HY, Sung R, Kim YC, Choi W, Kim H, Lee G, et al. Regional distribution of interstitial cells of Cajal (ICC) in human stomach. Korean. J Physiol Pharmacol. 20I0;I4:317-24.

28. Bhaskaran K, Douglas I, Forbes H, Dos Santos Silva, Leon D, Smeeth L, et al. Body-mass index and risk of
22 specific cancers: A population-based cohort study of 5.24 million UK adults. Lancet. 20I4;384:755-65.

29. Miettinen M, Sobin LH, Lasota J. Gastrointestinal stromal tumors of the stomach: A clinicopathologic, immunohistochemical, and molecular genetic study of 1765 cases with long-term follow-up. Am J Surg Pathol. 2005;29:52-68.

30. Casali PG, Jost L, Reichardt P, Bielack S, Bjerkehagen B, Bonvalot S, et al. ESMO Guidelines Working Group. Gastrointestinal stromal tumours: ESMO clinical recommendations for diagnosis, treatment and follow-up. Ann Oncol. 2009;20(Suppl.4):64-7.

3I. Huang Z, Li Y, Zhao H, Zhao JJ, Cai JQ. Prognostic factors and clinicopathologic characteristics of small gastrointestinal stromal tumor of the stomach: A retrospective analysis of 3 I cases in one center. Cancer Biol Med. 2013;IO:I65-8. 